\title{
Éléments pour une sémantique de ON
}

\author{
Lise Hamelin \\ LT2D, Université de Cergy-Pontoise, France \\ Lise.hamelin@u-cergy.fr
}

Résumé. Les études consacrées au pronom on se fixent en général pour objectif, soit de rendre compte du fonctionnement textuel, discursif, énonciatif, du marqueur, soit de rendre compte des possibilités de traduction de on par la mise en contraste avec d'autres systèmes linguistiques et/ou l'étude de la traduction. Ces approches ont livré des apports indiscutables, sur lesquels nous reviendrons rapidement. Elles illustrent des démarches et des méthodologies différentes mais ont en commun le fait qu'elles posent la question de l'identité de on dans la perspective d'une problématique référentielle. Dans ces pages, nous proposons d'envisager ce pronom sous un autre angle, celui de son fonctionnement, de son comportement en contexte. S'interroger sur ce que fait on, c'est aussi se demander ce qui fait on, et proposer un autre regard sur ce pronom. On laisse de côté les approches diachroniques du pronom, nous fixant comme objectif de rendre compte de son fonctionnement en synchronie. En effet, dans ces pages, nous envisageons ce pronom sous l'angle de son fonctionnement, de son comportement en contexte. S'interroger sur ce que fait on, c'est aussi se demander ce qui fait on, et proposer un autre regard sur ce pronom. Plus précisément, nous postulerons que on est la trace d'une opération énonciative particulière, définitoire, dont la mise en œuvre va générer en contexte différents effets de sens (pudeur, hypocoristique, distanciation) et se satisfaire d'une grande variété de contenus référentiels. On s'appuiera sur un corpus d'énoncés attestés récents (textes français des années 2010 issus essentiellementde FRANTEXT et occurrences tirées du web) sur lesquels on procédera à des manipulations pour montrer que l'emploi de on, en particulier lorsque son référent est aisément identifiable, témoigne d'un refus de prise en charge d'énonciative et/ou d'un refus d'implication du locuteur dans l'événement dont il rend compte, d'une part; et, d'autre part, que l'emploi d'un tel marqueur déstabilise la relation prédicative de telle manière qu'elle accorde un statut particulier aux propriétés du prédicats, qui se trouvent mises en valeur.

\begin{abstract}
So far, works on the French pronoun on have focused on two major goals : accounting for the functioning of on, as far as the discours and stylistic aspects are concerned or accounting for the différent possibilites available to translate this pronoun in other languages. These approaches have provided valuable results and observations, which will be reminded to the reader. They resort to different theoretical backgrounds and tools but share one common purpose : report on the referential construction of on.
\end{abstract}


Here, I would like to try another angle by paying particular attention to the pronoun's behaviour and functioning in relation to the context in which it appears. Wondering what on does is also questioning what on is and proposing another approach to the problem. More acurately, I will present a hypothesis according to which on marks a particular enunciative operation that is its modus operandi, and the way this operation interacts with the context triggers the different senses of the pronoun (reserve, modesty, affection). This work relies on examples taken from French novels from the 2010s, and occasional occurrences from the world wide web. These examples will be altered to test our hypothesis which is the following : on denotes a refusal from the utterer and or the speaker to get involved in the process they are refering to, on the one hand, and, on the other hand, on unbalance the predicative relation, and thus, gives particular importance to the properties of the predicate at the expense of the subject.

Les études consacrées au pronom on se fixent en général un objectif parmi les suivants :

- $\quad$ soit elles rendent compte du fonctionnement textuel, discursif, énonciatif, du marqueur. C'est le cas, entre autres, des travaux conduits dans le cadre de la ScaPoLine par Fløttum, Jonasson et Norén (2007) mais aussi des études de Muller 1979, Atlani 1984, Boutet 1986, Violett 1988, Rabatel 2001, qui s'attachent avant tout à clarifier la question de la référence de $o n$.

- $\quad$ soit elles rendent compte des possibilités de traduction de on par la mise en contraste avec d'autres systèmes linguistiques et/ou l'étude de la traduction (François 1984, Guillemin-Flescher 1981, Boutet 1986, Viollet 1988, Tamba 1989, Skibinska 2004, Tartarin 2013).

Ces approches ont livré des apports indiscutables, sur lesquels nous reviendrons. Elles illustrent des démarches et des méthodologies différentes mais ont en commun le fait qu'elles posent la question de l'identité de on dans la perspective d'une problématique référentielle. On laisse de côté les approches diachroniques du pronom, nous fixant comme objectif de rendre compte de son fonctionnement en synchronie. En effet, dans ces pages, nous envisageons ce pronom sous l'angle de son fonctionnement, de son comportement en contexte. S'interroger sur ce que fait on, c'est aussi se demander ce qui fait on, et proposer un autre regard sur ce pronom. Plus précisément, nous postulerons que on est la trace d'une opération énonciative singulière, dont la mise en œuvre va générer en contexte différents effets de sens (pudeur, hypocoristique, distanciation) et se satisfaire d'une grande variété de contenus référentiels.

\section{Qu'est on ?}

Les travaux consacrés à on font état d'un comportement versatile du marqueur, illustré jusque dans les intitulés de ces écrits (pronom à facettes, chez Fløttum, Jonasson et Norén 2007, illusionniste, chez Atlani 1984, doublement énigmatique chez Tamba 1989 : «la double énigme de on »). On, rappelons-le, occupe toujours la fonction syntaxique de sujet, il est parfois commutable avec un pronom personnel, parfois avec un pronom indéfini, il peut renvoyer à un référent singulier ou pluriel et inclure, ou non, l'énonciateur. Sa référence peut être générique ou spécifique, quoi qu'il en soit, elle est entièrement déterminée par le contexte d'apparition du pronom. Ainsi, Atlani 1984 présente on comme un pronom n'ayant « aucune valeur référentielle », nous informant uniquement du caractère humain de son référent.

Cette plasticité de on est prise en considération et synthétisée dans un tableau proposé par Fløttum, Jonasson et Norén (2007). Nous reproduisons ce tableau ci-après en 
l'agrémentant d'énoncés empruntés à ces auteurs pour illustrer chaque cas de figure :

Tableau 1 : D’après Fløttum, Jonasson et Norén, 2007

\begin{tabular}{|l|l|l|l|}
\hline Indéfini & Pérsonnel \\
\hline Impersonnel & Générique & Spécifique & Neutre \\
\hline $\begin{array}{l}\text { Il, ce } \\
\begin{array}{l}\text { On était le 15 oc- } \\
\text { tobre }\end{array}\end{array}$ & On peut toujours rêver & On frappe à la porte & Nous \\
\hline
\end{tabular}

Ce tableau, on le voit, illustre la capacité de on à commuter avec toutes les personnes, sans autre contrainte que de référer à un ou plusieurs animé(s) humain(s). On ne peut prendre la place de il impersonnel (il y a, il faut, il importe de, ou encore il pleut) :

(1) * on y a, on faut, on importe de, on pleut

Certains y voient une conséquence directe de l'origine étymologique du pronom. On est, rappelons-le, dérivé de homo (Ramat et Sansò, 2011) au cas nominatif en latin.

Ruppli (1988) relève une autre contrainte d'emploi du pronom : il ne peut être effacé dans une coordination ou une juxtaposition.

«[...] l'indéfini on qu'il réfère à un animé indéterminé ou qu'il se substitue à un pronom personnel (1ère, 2ème ou 3ème personne) est toujours repris et employé devant chaque prédicat verbal coordonné. »

Ce phénomène peut être illustré par l'exemple (2), dans lequel la référence de on peut être identifiée comme un collectif incluant l'énonciateur :

(2) On paie nos impôts séparés, mais on a la même chambre à coucher. (FRANTEXT)

(2') * On paie nos impôts séparés, mais a la même chambre à coucher.

En (2), les deux occurrences de on sont coréférentielles et font l'objet d'un accord verbal de troisième personne du singulier, mais la première est reprise par un possessif de première personne du pluriel (nos). Ce possessif pourrait être considéré comme un obstacle à l'omission du sujet dans la proposition coordonnée. Toutefois, cette omission est problématique aussi dans un énoncé comme (3') :

(3) [...] ils se réunissent autour des bancs, ils discutent, boivent des canettes [...] (FRANTEXT)

$\left(3^{\prime}\right) *$ [...] on se réunit autour des bancs, discute, boit des canettes [...]

(3") [...] on se réunit autour des bancs, on discute, on boit des canettes [...]

En (3'), on a proposé de remplacer ils par on, ce qui ne pose aucune difficulté dans la première partie de l'énoncé mais est incompatible avec l'omission dans la seconde partie. (3'), dans lequel on a rétablit on comme sujet du prédicat boire des canettes est, en revanche, parfaitement acceptable.

Il semble toutefois que cette contrainte connaisse des exceptions, même si celles-ci sont très littéraires. Dans Le bon usage, Grévisse $(1986,1141)$ relève quelques exemples d'omission de on dans la proposition coordonnée, dont (4) (tiré de Sartre, Les Mots) :

(4) On retourna son portrait contre le mur et fit défense de prononcer son nom. (FRANTEXT) Nos informateurs refusent toutefois cet énoncé, de même que (2') et (3'), ce qui nous conduit à penser que la contrainte de répétition reste importante et à considérer l'omission de on dans la proposition coordonnée en (4) comme un procédé stylistique.

Un autre trait caractéristique du comportement de on nous est fourni par l'analyse contrastive, qui montre que ce pronom est, dans les langues germaniques en tout cas, souvent traduit par une diathèse passive, même en allemand et en suédois dans lesquels il existe un pronom « équivalent », man (Guillemin-Flescher 1984 pour l'anglais, François 1984 et Cabredo Ofherr 2008 pour l'allemand, Jonasson 2003 pour le suédois). La diathèse passive, rappelonsle, via la mise en œuvre d'une autre organisation de l'énoncé, thématise le complément d'objet. Elle octroie donc un statut particulier, une saillance à ce qui aurait constitué, dans un énoncé à la voix active, un élément du prédicat. 
$\mathrm{Au}$ vu de ces analyses et de l'identification de ces contraintes, il est possible d'apporter à la question qu'est on? les éléments de réponse récapitulés ci-dessous (pour une analyse plus détaillée de ces contraintes, voir Landragin et Tanguy, 2014) :

Tableau 2 : Les contraintes et spécificités d'emploi de on

\begin{tabular}{|l|ll|}
\hline \multirow{4}{*}{ ON } & - & est un pronom sujet \\
\cline { 2 - 3 } & - & renvoie à un animé ou un groupe d'animés humains \\
\cline { 2 - 3 } & $\begin{array}{l}\text { voit sa référence entièrement conditionnée par le co-texte, celle-ci peut-être } \\
\text { générique ou spécifique }\end{array}$ \\
\cline { 2 - 2 } & $\begin{array}{l}\text { fait difficilement l'objet d'une ellipse en cas de coordination ou de } \\
\text { juxtaposition de propositions à sujets coréférentiels }\end{array}$ \\
\cline { 2 - 3 } & $-\quad$ est souvent traduit par une diathèse passive dans les langues germaniques \\
\hline
\end{tabular}

\section{Que fait on ?}

Afin d'identifier les spécificités du fonctionnement de on, nous mettrons en œuvre les outils développés dans le cadre de la Théorie des Opérations Prédicatives et Énonciatives de Culioli (PLE 1, 2 et 3, 1990-1999).

\subsection{Outils théoriques}

On constitue le sujet d'une relation prédicative $\langle a r b>$ dans lequel $a$ correspond au terme source de la relation, ici le pronom sujet on, $r$ au relateur, le verbe, et $b$ au terme cible de la relation, à ce qui délimite le prédicat, et est le plus souvent instancié par les compléments essentiels du verbe. On implique que l'on a affaire à un type particulier de terme source $a$, dans la relation que celui-ci entretient avec le groupe prédicatif $r b$. $R b$ renvoie à une notion de procès, dont le groupe prédicatif contextualisé et affecté de marques aspectuo-temporelles constitue une occurrence, c'est-à-dire un exemplaire singulier (c'est sa dimension qualitative) occupant une portion d'espace-temps donné (c'est sa dimension quantitative). Ajoutons pour achever de fixer les idées que la TOPE voit les énoncés comme des agencements de termes repérés les uns par rapport aux autres, le repère originel étant le couple énonciateur $\left(\mathrm{S}_{0}\right)$ moment d'énonciation $\left(\mathrm{T}_{0}\right)$. Le sujet grammatical du verbe $\left(\mathrm{S}_{2}\right)$ est repéré par rapport au locuteur $\left(\mathrm{S}_{1}\right)$, la personne qui produit le discours, lui-même repéré par rapport à l'énonciateur, $\left(\mathrm{S}_{0}\right)$, la personne qui prend en charge le discours (celle qui assume les jugements d'existence et de valeur, le choix des notions convoquées, etc.). De fait, le sujet de l'énoncé se construit selon la formule suivante: $S_{2} \underline{\epsilon} S_{1} \underline{\epsilon} S_{0}$, qui se lit « le sujet de l'énoncé est repéré par rapport au locuteur, qui est repéré par rapport à l'énonciateur ».

Dans la TOPE, l'opération de repérage peut prendre trois valeurs : l'identification (c'est la même chose), la différenciation (c'est l'altérité faible, pas vraiment, pas tout à fait la même chose) et la rupture (c'est l'altérité forte, tout à fait autre chose). Ces trois opérations sont notées, respectivement $:=, \neq, \omega$. L'identification de $\mathrm{S}_{2}$ à $\mathrm{S}_{1}\left(\mathrm{~S}_{2}=\mathrm{S}_{1}\right)$ correspond au cas dans lequel le sujet grammatical est identifié au locuteur et est donc un sujet de première personne, la différenciation $\left(S_{2} \neq S_{1}\right)$, c'est la deuxième personne, la rupture $\left(S_{2} \omega S_{1}\right)$, c'est la troisième personne. On, comme le rappellent Bouscaren et Chuquet (1987), est susceptible de réaliser ces trois opérations, dans la mesure où il peut commuter avec toutes les personnes. Le type de repérage dont il est la trace est noté comme suit : $\mathrm{S}_{2} * \mathrm{~S}_{1}$, dans lequel * correspond à l'indétermination de l'opération de repérage. A notre sens, cette représentation n'est pas pleinement satisfaisante dans la mesure où, finalement, elle ne livre pas d'information sur le modus operandi de on et ne permet d'appréhender sa valeur que lorsqu'il est la trace d'un 
brouillage énonciatif, dans certains procédés stylistiques, ou lorsque la source de l'information est tue, dans un contexte journalistique ou de simple commérage. Les analyses qui traitent on dans le cadre de la polyphonie ne sont guère plus éclairantes en ce qu'elles dépassent rarement le niveau de la description.

\subsection{Hypothèses d'analyse}

A vrai dire, il ne nous parait pas impossible de traiter on comme un pronom de troisième personne, dans la mesure où il appelle un accord verbal de troisième personne du singulier. Le français est une langue accusative, dans laquelle le verbe s'accorde avec son sujet. La troisième personne est aussi l'accord verbal attendu derrière le pronom mann en allemand. En espagnol, il n'y a pas d'équivalent strict au pronom on mais une forme constituée du pronom réfléchi se et du verbe conjugué la troisième personne du pluriel permet de renvoyer à un sujet dont la référence est floue ou inconnue. Cet accord verbal est peut-être un choix par défaut, il n'empêche qu'il s'agit tout de même du choix retenu parmi l'ensemble des possibilités disponibles par le français mais aussi par d'autres langues possédant des équivalents de on. Nous associerons donc ce pronom au mode de repérage suivant: $S_{2} \omega S_{1}$.

On pourrait formuler deux objections principales à notre proposition d'analyse, la première est l'existence, selon certains linguistes d'une valeur impersonnelle de on (par exemple chez Fløttum, Jonasson et Norén 2007, cf. tableau 1 supra). Nous récusons l'existence d'une telle valeur : nous n'en avons trouvé aucun exemple dans lequel on ne puisse pas commuter avec nous. Ainsi, à la place de On était le 15 octobre dans le tableau 1, on pourrait aisément dire Nous étions le 15 octobre. Par ailleurs, on ne peut pas commuter avec il impersonnel ( $c f$. (1) supra). Il ne peut pas non plus commuter avec ce/ceci/cela : C'était un bon concert. $/ *$ On était un bon concert.

La seconde objection à une analyse de on comme pronom de troisième personne du singulier et la possibilité qu'il fasse l'objet d'une reprise par un autre pronom personnel (possessif, réfléchi). C'est vrai, uniquement pour la première personne du pluriel, comme on le voit en (5) et en (2) supra:

(5) On est nous-mêmes, c'est tout. (FRANTEXT) / On est soi-même, c'est tout.

(5') * On est moi-même/toi-mêmeleux-mêmes/lui-mêmelelle-même.

On reviendra un peu plus loin sur la relation privilégiée qu'entretiennent on et nous, en prenant en considération les remarques de Benveniste sur la référence des personnes plurielles. Nous verrons que la possibilité de faire commuter on et nous ne suffit pas à remettre en cause l'hypothèse d'analyse défendue dans ces pages.

S'en tenir là, cependant, impliquerait qu'il n'existe pas de différence entre on et les pronoms personnels de troisième personne $i l$ et elle, or, tel n'est pas le cas. Ce qui semble être la particularité de on, c'est qu'il pose d'emblée l'existence d'une rupture entre $\mathrm{S}_{1}$ et $\mathrm{S}_{0}$. Le on académique, le on journalistique ainsi que le on de «la rumeur » permettent à $\mathrm{S}_{1}$ d'indiquer qu'il n'est pas l'énonciateur du propos qu'il reprend. Nous défendons donc l'hypothèse de représentation suivante : $S_{2} \omega S_{1} \omega S_{0}$, soit on implique que le sujet de l'énoncé est en rupture avec le locuteur, lui-même en rupture avec l'énonciateur. Cette proposition de représentation ne permet pas de déduire a priori le type de relation dans lequel $\mathrm{S}_{2}$ se trouvera avec $\mathrm{S}_{0}$, et l'une des particularités de on est justement de pouvoir inclure ou non $\mathrm{S}_{0}$.

Enfin, il est peu souvent remarqué que le flottement sur le terme source de la relation prédicative entraîne une saillance plus importante du relateur et du terme cible $(r b)$, notamment dans les contextes non-assertifs, où le prédicat fait très souvent l'objet d'une valuation (ça ne se fait pas, c'est n'importe quoi, c'est surprenant/bizarre/mignon). Cette mise en valeur du prédicat est sans doute l'un des éléments qui permettent d'expliquer la grande fréquence des traductions de on par des énoncés à la voix passive, notamment dans les langues germaniques. 


\section{On pronom de troisième personne}

\subsection{On et la troisième personne en contexte non-assertif}

On est susceptible, comme il a été mentionné plus haut, de commuter avec n'importe quel pronom personnel lorsqu'il renvoie à un référent défini. Nous y voyons une conséquence du double repérage en rupture que nous proposons comme représentation du marqueur. $\mathrm{Si}_{2} \omega$ $S_{1} \omega S_{0}$, alors $S_{2}$ peut inclure l'énonciateur $\left(S_{2}=S_{0}\right)$ et/ou le co-énonciateur $\left(S_{2} \neq S_{0}\right)$ ou bien exclure l'énonciateur et le co-énonciateur $\left(S_{2} \omega S_{0}\right)$, autrement dit, si le locuteur se trouve en rupture avec l'énonciateur et le sujet de l'énoncé, la relation entre ces deux termes est sousspécifiée, ce qui explique le caractère non stabilisé de la référence de on (inclusif, exclusif ou flou selon les termes de Landragin et Tanguy 2014). Ce dernier cas de figure est illustré en (6) :

(6) [...] une cousine de ma grand-mère s'indigne que la France puisse subir les mots d'ordre d'un petit Juif étranger. Je la regarde avec stupeur : je ne savais pas qu'on puisse en être encore là, vingt-cinq ans après l'Occupation.(FRANTEXT)

En (6), les pronoms la et on sont coréférentiels, ils renvoient tous deux à une cousine de ma grand-mère. On peut donc imaginer remplacer on par elle dans l'énoncé, et on constate que la substitution appelle une modification de la forme du verbe en (6') :

(6') [...] je ne savais pas qu'elle pouvait/? puissel? pût en être encore là, [...]

Il existe une différence notable entre $\langle a-r b\rangle$ avec on comme terme $a$ et $\langle a-r b\rangle$ avec elle comme terme $a$, puisque l'on a dû, pour opérer la substitution, passer du mode subjonctif au mode indicatif, et ce faisant, de la mise en cause de l'existence d'un événement /X-en être encore là/, quel que soit $\mathrm{X}$, à une interrogation sur les propriétés du référent de elle. (6') suppose que l'événement entre en conflit avec une représentation que l'énonciateur aurait eu préalablement du référent de elle. En d'autres termes, le fait de substituer elle à on a réduit la référence du terme source et stabilisé la relation prédicative. La possibilité de commutation n'implique donc pas la stricte équivalence entre les deux pronoms. La reprise du référent de la par on en fait un individu représentatif d'un groupe susceptible de valider la relation prédicative, pour invraisemblable qu'elle soit (je ne savais pas que). On a une portée généralisatrice, par conséquent, le substitut le plus approprié pour on dans ce contexte serait sans doute un syntagme indéfini qui que ce soit, :

(6") [...] Je la regarde avec stupeur : je ne savais pas que qui que ce soit puisse/pût en être encore là, vingt-cinq ans après l'Occupation.

En (6"), l'emploi du subjonctif (qui que ce soit) accentue le caractère instable du terme $a$, et donc de la relation prédicative qu'il est censé validé. Cette instabilité a des conséquences sur le mode de prise en charge de la relation prédicative, avec notamment le passage au premier plan des propriétés notionnelles associées au prédicat, de ce qu'il véhicule en termes de représentation en tant qu'occurrence d'une notion de procès particulière, c'est-à-dire ce qui relève de la délimitation qualitative de cette occurrence. (6) livre une interprétation selon laquelle aujourd'hui, il ne devrait exister aucun $a$ à même de valider en être encore là, compte tenu des propriétés du prédicat (« c'est inacceptable », « inconcevable », « inadmissible »). Cette évaluation repose sur des termes du co-texte immédiat : là démonstratif pouvant être associé à une valuation négative, encore, qui suppose qu'on se soit intéressé, au moins virtuellement, à un état de faits autre que /en être là/, état de faits supposé attendu vingt-cinq ans après l'Occupation, le verbe modal pouvoir, qui est pour beaucoup dans l'émergence de l'interprétation selon laquelle on ne devrait pas trouver d'occurrence de la relation prédicative validée, sans oublier le fait que tous ces éléments s'inscrivent dans une complétive tombant sous la portée du verbe savoir affecté de le négation. (6) fait intervenir de nombreux marqueurs d'évaluation et de valuation qui contribuent à faire passer au premier plan les propriétés notionnelles de l'occurrence de procès.

A notre connaissance, il est rarement mentionné que on en contexte négatif peut 
commuter avec personne, avec, cependant, une variation sémantique qui n'est pas sans rappeler la différence observée plus haut entre (6) et (6') :

(7) On ne peut pas demander d'olives à un figuier.(FRANTEXT)

(7') Personne ne peut demander d'olives à un figuier.

Comme en (6), avec le verbe modal pouvoir, on retombe sur un processus d'évaluation des chances de venue à l'existence de l'événement dénoté par la relation prédicative. Alors que (7') peut être compris comme signifiant «il n'existe aucun individu possédant les compétences requises pour demander à un figuier de produire des olives ». Ce n'est pas ce que l'énoncé (7) signifie : il met en avant le caractère absurde, irréalisable, de la notion de procès indépendamment des propriétés d'un éventuel sujet-valideur : demander à un figuier de produire des olives, ça n'a pas de sens.

L'emploi de on permet d'énoncer des principes fondamentaux et irréfutables :

(8) «On ne parle pas comme ça à sa mère. » (https://dominiquedimey.com/home/dominiquedimey-et-lenfance-on-en-parle-dans-la-presse-et-les-medias/)

(8) pourrait être paraphrasé par « Parler comme ça à sa mère, cela ne se fait pas », dans lequel cela reprend le prédicat et explicite l'idée selon laquelle ce sont les propriétés même de la notion de procès qui rendent sa venue à l'existence inenvisageable. Notons que la possibilité de substituer personne à on en contexte négatif est liée au caractère générique de l'énoncé (8), remarque qui rejoint les observations de P. Cadero Hofherr à propos des lectures épisodiques des énoncés mettant en jeu le pronom on :

«En effet, avec des passifs et des inaccusatifs, on ne permet pas de lectures épisodiques (Cinque 1988, voir aussi Legendre 1989). Comme le montrent les exemples, la même généralisation est valable pour le man allemand (et pour le man danois et suédois également, voir Egerland 2003).

[8]a ?Gestern wurde man verprügelt.

Hier on a été tabassé.

(la lecture "qqn a été tabassé" est impossible)

[8]b ?Gestern kam man um10 Uhr an.

Hier on est arrivé à $10 \mathrm{~h}$.

(la lecture "qqn est arrivé à $10 \mathrm{~h} "$ est impossible) »

Dans les énoncés négatifs comme (6), (7) et (8), la remise en cause de l'existence d'une occurrence de la relation prédicative repose non sur l'existence d'un sujet compétent pour valider la relation prédicative mais sur une forme d'évaluation du prédicat. On peut y commuter avec un pronom de troisième personne ou un pronom indéfini comme personne, sa référence ayant une importance minime puisque c'est le caractère invraisemblable, impossible, inadmissible du prédicat qui est mis en avant. De fait, le choix du pronom on est aussi une prise de position énonciative puisqu'il s'agit de faire passer au premier plan l'évaluation ou la valuation de la relation prédicative validée par un terme $a$, quel qu'il soit.

\subsection{On et la troisième personne en contexte assertif positif}

Dans des contextes positifs, avec des procès au mode indicatif, on ne fonctionne pas différemment, mais le fait que l'existence du prédicat ne soit pas mise en question génère des effets de sens autres, comme on peut le voir en (9) :

(9) Ils jouent à l'endurci mais ils n'ont encore rien vu des saletés. On est au repos, bien tranquille à bouffer son pilon de poulet, bombance et rigolade, une bombe sous le cul.(FRANTEXT)

On est ici coréférentiel avec ils, qui peut lui être substitué :

(9') Ils jouent à l'endurci mais ils n'ont encore rien vu des saletés. Ils sont au repos, bien tranquilles à bouffer leur pilon de poulet, bombance et rigolade, une bombe sous le cul.

On remarque que l'énoncé dans lequel on apparaît est inséré dans une proposition explicitant 
l'idée véhiculée dans la phrase précédente : l'enjeu consiste ici, pour l'énonciateur, à préciser ce qu'il entend par /jouer à l'endurci/, qui correspond pour lui à /être au repos, bien tranquille à bouffer son pilon de poulet, bombance et rigolade, une bombe sous le cul/, etc. L'ancrage existentiel d'une occurrence de lêtre au repos $[\ldots] /$ ne fait pas débat, dans la mesure où celleci est assimilée à l'occurrence de /jouer à l'endurci/, qui possède une délimitation quantitative, les deux procès sont d'ailleurs au présent de l'indicatif. Dans cet exemple, la relation dont on est le terme source (on est au repos [...] sert de délimitation qualitative au prédicat de la phrase précédente (/n'avoir encore rien $v u /)$.

On observe aussi une prééminence du qualitatif en (10) :

(10) Il avait des hommes, des soldats; il envoyait ses soldats à travers la campagne voler des enfants de paysans. On lui ramenait ces enfants et il les égorgeait dans des buts de magie. (Michel Tournier, Lettres parlées à son ami allemand Hellmut Waller (1967-1998), Gallimard, 2015)

En (10), On est coréférentiel avec ses soldats : ce sont eux qui ramènent les enfants :

(10') [...] L/Ses soldats lui ramenaient ces enfants et il les égorgeait dans des buts de magie. En (10), On lui ramenait ces enfants explicite, justifie il envoyait ses soldats [...] voler des enfants et correspond à sa justification notionnelle, à sa raison d'être.

C'est encore le qualitatif qui passe au premier plan dans un exemple comme (11), assez proche des emplois génériques de on que nous aborderons dans le paragraphe suivant: (11) En Creuse, on invente, on se décarcasse, on crée pour améliorer le bien-être des seniors (janvier 2017). (https://www.lamontagne.fr/gueret/sante-medecine/2017/01/08/en-creuseon-invente-on-se-decarcasse-on-cree-pour-ameliorer-le-bien-etre-des-

seniors_12233964.html)

(11') Les creusois inventent, se décarcassent, créent pour améliorer le bien-être des seniors. En (11'), la substitution de les creusois à on permet l'ellipse du sujet dans les propositions juxtaposées suivant la première. L'accumulation de procès ne correspond pas à une succession d'actions mais à un ajustement qualitatif avec le renvoi à des notions de procès quasi-synonymes ou s'explicitant les unes les autres. D'ailleurs, même lorsque l'on a affaire à une succession d'événements dynamiques, ceux-ci ne sont pas qualitativement individués, dans la mesure où ils sont toujours repérés par rapport à une classe de situations dont la première occurrence de procès est souvent l'étalon. A titre d'exemple, une recherche sur google.fr, sans limitation de domaine, avec la syntaxe « on franchit la $*$ on » ne livre que des occurrences au présent de l'acabit de (12) et (13) :

(12) Quand on franchit la ligne d'arrivée, on peut poursuivre les dégustations. (http://tva.canoe.ca/emissions/salutbonjour/chroniques/detail/la-colombie-britannique)

(13) Lorsque l'on franchit la porte du vestiaire, on oublie les soucis de la vie extérieure : toute l'attention doit être consacrée à la pratique. (règlement intérieur d'un club d'Aïkido) Pour lever l'ambiguïté, puisque on franchit peut être aussi bien un présent qu'un passé simple de l'indicatif, on a lancé une requête avec la syntaxe «on passa la * on », qui n'a livré aucune occurrence. Une recherche avec « on a franchi la $*$ on » livre peu de résultats et ceux-ci sont comparables à (12) et (13). Ainsi, même associé à une série de verbes dynamiques, on ne se combine pas avec l'expression d'une succession d'actions. Précisons toutefois que on peut tout à fait s'utiliser avec un renvoi à un événement singulier comme en (14), mais si celui-ci est le sujet de plusieurs procès, c'est nécessairement la dimension qualitative de l'occurrence qui prend le pas sur sa dimension quantitative, c'est-à-dire qu'il s'agit davantage de savoir de quoi il retourne que de savoir si l'événement a effectivement eu lieu.

(14) Attendre, encore et toujours. Nous devrions être fixés sur notre sort d'ici quelques heures. On nous a prescrit de consulter les panneaux d'affichage à l'accueil [...] (FRANTEXT)

Nous voyons dans ce phénomène une conséquence du caractère non stabilisé de la référence de on, qui aboutit à la mise en avant non pas de l'ancrage existentiel de l'événement dont il est le sujet mais des propriétés de cet événement. En ce sens, (12) et (13) sont proches des cas dans lesquels on a un fonctionnement générique. 


\subsection{Les emplois génériques de on}

Dans les emplois génériques de on, l'identité du sujet de la relation prédicative n'importe en rien. On y met en avant l'aspect qualitatif, les propriétés fondamentalement associées à la notion de procès. On le rencontre souvent dans les subordonnées de comparaison, comme en (15) :

(15) [...] il aurait fallu la soulever, la porter sur son lit et border son drap, comme on le fait aux petits enfants, mais aucun ne l'aurait tenté.(FRANTEXT)

Il s'agit bien ici d'évoquer la manière dont les petits enfants sont en principe bordés sans se préoccuper de l'existence avérée d'une quelconque occurrence de la notion de procès / border les petits enfants/, notion qui n'est mobilisée dans l'énoncé qu'en tant que représentation servant de repère à la comparaison.

La substitution d'un groupe nominal générique pluriel à on donnerait le sentiment d'une caractérisation fondée sur l'observation d'un usage, d'un comportement habituel d'un groupe dans une classe de situations, interprétation qui n'est pas donnée dans l'énoncé de départ, même si elle peut être reconstruite par inférence :

(15') [...] il aurait fallu la soulever, la porter sur son lit et border son drap, comme les gens/les parents/les adultes le font aux petits enfants, mais aucun ne l'aurait tenté.

Ce phénomène est évidemment lié au fait qu'en remplaçant on par un groupe nominal, même générique (les gens), on a rattaché l'événement dénoté par le prédicat à une catégorie d'individus. Il ne s'agit plus d'aborder la notion/border les enfants/ pour ses propriétés pures, mais dans la mesure où elle est associée à un groupe (thématisé dans l'énoncé) dont elle constitue une propriété. Il n'est guère surprenant que on soit souvent traduit, dans les langues qui ne disposent pas d'un tel pronom, par une diathèse passive, dans laquelle c'est le constituant qui aurait en principe occupé la position d'objet, c'est-à-dire un élément du prédicat, qui se trouve thématisé.

Il n'y a rien d'étonnant non plus à ce que on puisse être employé pour évoquer la rumeur populaire avec les verbes introducteurs de discours rapporté, dans la mesure où le fait qu'il y ait du « dit» et la question du contenu de ce « dit » importent bien davantage que l'identité de ceux qui colportent le bruit. Se moquer du qu'en dira-t-on, c'est bien n'attacher aucune importance au «dit» en tant que produit d'une activité, peu importe qui « dit» effectivement.

\subsection{On sujet d'un verbe de perception}

L'analyse de on appelle un commentaire sur les valeurs de ce pronom lorsqu'il est associé à un verbe de perception. Ces emplois ont été commentés par Rabatel (2001), mais aussi par Maingueneau (2000), pour qui on est à même de renvoyer à un individu identifié comme un «membre quelconque d'une collectivité ». La collectivité en question est alors construite dans le contexte, notamment, par le biais d'un complément circonstanciel. En (16), c'est la localisation dans l'espace exprimée par de cette forteresse imposante qui permet de construire la classe des individus auxquels on est susceptible de référer : l'activité de perception dénotée par $<\mathrm{a}-$ distinguer les maisons protestantes des catholiques à la forme de leurs cheminées $>$ est accessible à n'importe quel terme $a$ dont la localisation est assimilable à de cette forteresse imposante :

(16) De cette forteresse imposante, dont les terrasses à degrés, blotties en coquille d'escargot, dominent le Gardon et le village, on distingue encore les maisons protestantes des catholiques à la forme de leurs cheminées; en montant sur le donjon en ruine, c'est toute la vallée du Rhône qu'on découvre.

Rabatel (2001) montre que la perception peut être attribuée à un personnage particulier du récit, à un sujet-point de vue focalisé, comme en (17), qui lui est emprunté :

(17) Et sans que Fouchard s'y opposa, il [= Maurice] alla ouvrir la fenêtre. Toute la vallée 
noire se creusa, roulant sa mer de ténèbres. Pourtant, lorsque les yeux s'étaient habitués, ON distinguait très nettement le pont, éclairé par les feux des deux berges. [...] Il avait laissé la fenêtre ouverte.

A propos de (17), Rabatel (2001:29) indique que « c'est en effet Maurice, et lui seul, qui va se placer à la fenêtre, comme le soulignent, en ouverture et en clôture de cette pause descriptive focalisée, les expressions « il alla ouvrir la fenêtre »et «il avait laissé la fenêtre ouverte ». Il est tout à fait exact que le point de vue de Maurice est le point de vue focalisé dans le passage cité. Néanmoins, comme le souligne l'auteur en conclusion de cette même étude :

« Reste à expliquer le recours privilégié à une forme à l'interprétation si délicate. Si, en contexte narratif, « on» coréfère à un focalisateur explicite ou identifiable par inférences sur la base de la référenciation, pourquoi diable le refus de lever explicitement l'ambiguité ! C'est que « on» en dit plus qu'un simple pronom personnel. Sa valeur de base, indéfinie, n'est jamais totalement supprimée : soit que le locuteur veuille faire entendre que l'identification ne peut être plus précise ; soit, plus sûrement, qu'il veuille nous faire entendre qu'il ne souhaite pas l'être. » (2001:32).

Notre hypothèse d'analyse peut apporter des éléments de réponse à cette question. L'identité du sujet-point de vue n'a aucune importance dans ces contextes. Ce qui importe, c'est que le pont soit visible depuis la fenêtre, les maisons depuis cette forteresse imposante. C'est le prédicat qui passe au premier plan, et plus précisément ici, le terme cible, l'objet de la perception, : celui-ci est visible, observable pourvu que on occupe telle position spatiale. C'est l'objet de perception qui fait l'objet d'une description, ce qui est cohérent avec l'idée que finalement, on fait passer les propriétés du prédicat au premier plan, sans doute encore plus dans les exemples dans lesquels il est aisé de reconstruire sa référence : pourquoi utiliser on sinon? Un exemple comme (17) ne dit rien de moins que la chose suivante : c'est bien Maurice qui est sujet-percevant mais cela pourrait être n'importe qui d'autre, n'importe quel terme source pourvu que son référent occupe la position définie par le prédicat ouvrir la fenêtre.

On est donc un pronom de troisième personne, comme en témoigne l'accord verbal qui le suit, mais dont la référence n'est pas stabilisée. Cette référence peut être reconstruite grâce aux éléments du contexte de manière plus ou moins évidente. Quoi qu'il en soit, ce caractère instable du terme source de la relation prédicative met en question l'ancrage existentiel d'une occurrence effectivement validée de la relation et aboutit à la mise en avant des propriétés du prédicat (le relateur $r$ et le terme cible $b$ ). C'est la dimension qualitative qui prend le pas sur la dimension quantitative, d'où l'emploi de on dans des contextes de comparaison, des contextes génériques avec des repérages par rapport à une classe de situations, et l'abondance d'emploi traités traditionnellement comme stylistiques ou affectifs, comme nous nous apprêtons à le voir.

\section{La première et la deuxième personne}

\subsection{On et la première personne du pluriel}

La première chose qui vient à l'esprit lorsque l'on envisage la commutation de on avec d'autres pronoms personnels, c'est sa fréquente concurrence avec le pronom de première personne du pluriel nous. Cette concurrence est le plus souvent considérée comme stylistique (Fløttum, Jonasson et Norén (2007)) et attribuée au genre discursif dans lequel l'occurrence apparaît. On aurait vocation à se substituer à nous dans des genres oraux et/ou relâchés (d'après Fløttum, Jonasson et Norén (2007), ou encore Rey-Debove (2001)). N'ayant pas nous-mêmes mené d'étude statistique sur la répartition de on dans les genres textuels, nous nous alignerons sur cette réflexion qui coïncide avec les observations intuitives que nous pourrions formuler à partir de nos textes, en tout cas pour ce qui concerne les genres littéraires dans lesquels on 
apparaît comme substitut de nous dans le discours rapporté au discours direct.

D'un point de vue théorique, le recouvrement partiel de on et nous n'est pas en contradiction avec notre analyse de on comme pronom de troisième personne.

«Il est clair en effet que l'unicité et la subjectivité inhérentes à «je» contredisent la possibilité d'une pluralisation. S'il ne peut y avoir plusieurs « je » conçus par le « je » même qui parle, c'est que «nous » est, non pas une multiplication d'objets identiques, mais une jonction entre « je » et le « non-je », quel que soit le contenu de ce «non-je ». [...] La présence $\mathrm{du}$ « je » est constitutive du « nous ». » (Benveniste, PLG1 233)

Le dernier point souligné par Benveniste est particulièrement important : nous implique l'identification partielle de $S_{2}$ (le sujet de l'événement) avec $S_{1}$ (le locuteur) ainsi qu'un élément d'altérité. Nous peut donc englober la première personne et la seconde (« je »- »tu » ou « je »- »vous »), la première et la troisième (« je »- « il/elle » ou « je »- « eux/elles »), ou encore les trois (« je »- «tu/vous» - «il/elle/eux/elles »). En cela, nous contient en germe les mêmes possibilités que on, à ceci près qu'il inclut le locuteur $\left(\mathrm{S}_{2}=\mathrm{S}_{1}\right)$. S'appuyant sur cette proximité Rey-Debove (2001) en arrive même à émettre l'hypothèse selon laquelle on pourrait être amené à remplacer nous à l'oral.

La différence entre les deux pronoms étant ténue dans certains cas, on pourra s'intéresser aux contextes, oraux ou écrits dans lesquels ils coexistent et renvoient au même référent pour tenter de mettre en lumière les éléments qui permettent de les distinguer l'un de l'autre. Soient les trois exemples suivants tirés de romans contemporains :

(18) Surtout, vu son aptitude au mimétisme, on pouvait se réjouir (avais-je dit à Serguei) qu'il eût préféré l'Étoffe des héros à l'Empire contre-attaque, nous n'avions évidemment aucune envie d'être commandés par Dark Vador. (FRANTEXT)

(19) Je fis les derniers mètres pour le rejoindre, et on se donna l'accolade, avec précaution, mimant l'accolade plutôt que la donnant vraiment, il me tapa deux ou trois fois doucement dans le dos. Il s'empara de ma valise et on passa les portes [...] Nous ne disions rien sous les vastes auvents de verre incurvés de l'aéroport, et nous attendions la voiture qui devait venir nous cherche.(FRANTEXT)

Nous pensons que on, lorsqu'il renvoie à un référent identifié, permet de mettre en avant la nature du procès plus que l'agent qui l'effectue, et les propriétés des énoncés (18) à (19) semblent aller dans ce sens. En (19), c'est on qui est utilisé pour caractériser la manière dont les référents de on/nous se saluent et l'énoncé comporte un certains nombres d'ajustements qualitatifs qui permettent de mieux décrire ce qui se produit (avec précaution, mimant l'accolade plutôt que la donnant vraiment, il me tapa deux ou trois fois doucement dans le $d o s$ ). Lorsque les procès sont qualitativement moins marqués, c'est nous qui est utilisé (Nous ne disions rien, nous attendions). En (18), la présence du verbe modal pouvoir à l'imparfait accentue la légitimité, le bien fondé de la venue à l'existence d'une occurrence de /se réjouir/ compte tenu des circonstances ( $v u$ son aptitude au mimétisme), et ce, quelle que soit l'identité sujet s'inscrivant dans le contexte. En revanche, pour parler de l'émotion effectivement éprouvée par les référent de on/nous, c'est nous qui est utilisé.

Nos textes comportent peu d'occurrences coréférentielles de on et nous, ainsi, on aura du mal à s'aventurer au-delà des quelques remarques ci-dessus, mais peut-être peut-on les mettre en regard avec certains résultats de Fløttum, Jonasson et Norén (2007).

En faisant passer au premier plan la délimitation qualitative de l'occurrence de procès (sa nature, ses propriétés), on relègue au second plan sa délimitation quantitative, c'est-à-dire la question de l'ancrage existentiel de l'événement, comme on a pu le voir. De leur côté, Fløttum, Jonasson et Norén (2007) ont évalué, à partir de leur corpus, la propension de on à se combiner avec telle ou telle forme temporelle du français, ce qui est particulièrement intéressant puisque certaines de ces formes permettent de mettre davantage en avant la délimitation qualitative ou quantitative de l'occurrence de procès. Nous empruntons le tableau ci-dessous à Fløttum, Jonasson et Norén (2007) : 
Tableau 3 (adapté de Fløttum, Jonasson et Norén (2007), Répartition du on sur les temps verbaux :

\begin{tabular}{|l|l|l|l|l|l|l|l|}
\hline & $\begin{array}{l}\text { Présen } \\
\mathrm{t}\end{array}$ & $\begin{array}{l}\text { Imparfai } \\
\mathbf{t}\end{array}$ & $\begin{array}{l}\text { Conditionn } \\
\mathrm{el}\end{array}$ & Futur & $\begin{array}{l}\text { Passé } \\
\text { simple }\end{array}$ & $\begin{array}{l}\text { Passé } \\
\text { composé }\end{array}$ & $\begin{array}{l}\text { Plus-que- } \\
\text { parfait }\end{array}$ \\
\hline Madame Bovary & $\begin{array}{l}136 / 2 \\
2 \%\end{array}$ & $\begin{array}{l}\mathbf{2 6 6 / 4 3 , 5} \\
\mathbf{\%}\end{array}$ & $40 / 6,5 \%$ & $9 / 1,5 \%$ & $\mathbf{9 4 / 1 5 \%}$ & $\mathbf{1 7 / 3 \%}$ & $29 / 5 \%$ \\
\hline Une vie & $\begin{array}{l}71 / 18 \\
\%\end{array}$ & $\mathbf{1 1 9 / 3 0 \%}$ & $32 / 8 \%$ & $\begin{array}{l}10 / 2,5 \\
\%\end{array}$ & $\mathbf{1 3 4 / 3 3 , 5 \%}$ & $\mathbf{1 0 / 2 , 5 \%}$ & $22 / 5,5 \%$ \\
\hline $\begin{array}{l}\text { La maison } \\
\text { assassinée } \\
\text { Moüra }\end{array}$ & $\begin{array}{l}48 / 23 \\
\%\end{array}$ & $\mathbf{1 3 6 / 3 3 \%}$ & $40 / 10 \%$ & $174 \%$ & $\mathbf{1 1 / 3 \%}$ & $\mathbf{5 0 / 1 2 \%}$ & $54 / 13 \%$ \\
\hline
\end{tabular}

On entretient des affinités particulières avec l'imparfait. Il est en revanche moins souvent utilisé avec les aoristes que sont le passé simple et le passé composé. Bien qu'il ne soit pas incompatible avec ces formes, celles-ci sont sous-représentées, que ce soit dans les textes du XIXème (Madame Bovary et Une vie) ou dans les textes du XXème (La maisons assassinée et Moïra). Dans nos textes des années 2010, le rapport entre les énoncés dans lesquels on est le sujet d'un verbe au passé simple, comme en (20), et ceux dans lesquels il est le sujet d'un verbe à l'imparfait est de 1/13.

(20) Il n'y a rien encore de prouvé. Nous allons faire une enquête. Puis, on nous conduisit au commissariat. Ce brave homme n'avait pas reçu d'ordres et ne savait que faire de nous. (FRANTEXT)

Le passé composé, en (22), est davantage représenté, avec un rapport 1/5.

(21) Après le déjeuner, on nous a réunis dans la salle de restaurant. (FRANTEXT)

Le fait que le passé composé entre aujourd'hui plus souvent en combinaison avec on que le passé simple tient en partie à sa plus grande fréquence absolue dans les textes contemporains. Par ailleurs, le passé composé s'emploie généralement dans un registre plus relâché que le passé simple, autrement dit, dans des conditions qui favorisent l'emploi de on (plutôt que celui de nous, par exemple).

Que la différence de registre explique en partie l'écart de distribution entre le passé composé et le passé simple avec on est indiscutable, néanmoins ces deux formes entrent nettement moins souvent en combinaison avec on que l'imparfait. Or, pour faire un raccourci un peu grossier, le passé simple et le passé composé mettent l'accent sur la délimitation quantitative de l'occurrence de procès en localisant l'événement dans le passé. Par conséquent, ces formes sont généralement associées à des événements actualisés, rattachés à un ancrage existentiel. L'imparfait, lui, n'est pas un aoriste et ne présente pas l'événement comme achevé, ce qui peut permettre d'insister sur ses propriétés intrinsèques. Pour Le Goffic (1986 : 55), l'imparfait est un non présent, mais il ne s'agit pas non plus d'un temps du passé. Cette remarque s'appuie sur l'ambiguïté d'exemples comme «Sans vous, je m'ennuyais. » qui, hors contexte peuvent donner lieu à une interprétation passée ou irréelle. Chez Guillaume (1994 (1951) :214-215) encore, l'imparfait est vu comme un passé décadent. En fait, ce qui permet de localiser l'événement du procès à l'imparfait dans le temps, ce sont les inférences que l'on peut tirer du repérage interpropositionnel. Autrement dit, la localisation dans le temps d'un procès à l'imparfait est déduite du contexte dans lequel il est employé.La fréquente combinaison de on avec cette forme temporelle nous paraît aller dans le sens de notre hypothèse selon laquelle on, en déstabilisant le terme source de la relation prédicative, mettrait en avant les propriétés du relateur et du terme cible, c'est-à-dire la délimitation qualitative du prédicat. 


\subsection{Remarques sur le discours scientifique}

Notre analyse peut aussi rendre compte de l'emploi de on dans le discours scientifique ou académique. Ce pronom y permet à l'auteur-énonciateur de mettre en avant le procès, qui correspond à ses hypothèses, ses découvertes, ses conclusions, en passant sous silence qu'il est à l'origine des analyses défendues. Celles-ci sont présentées comme si elles étaient susceptibles d'être prises en charge par n'importe qui ayant eu accès aux même données, ce qui leur confère, du moins en apparence, une plus grande objectivité. On relève alors d'une stratégie rhétorique qui permet à l'énonciateur d'éviter l'identification de $\mathrm{S}_{2}$ et $\mathrm{S}_{0}$, et par conséquent, de placer la relation prédicative sous la responsabilité virtuelle de n'importe quel énonciateur potentiel, comme illustré en (22) :

(22) «Tout au long des analyses freudiennes, on perçoit que le sujet se sert de la parole et du discours pour se "représenter" lui-même tel qu'il veut se voir, tel qu'il appelle l' " autre » à le constater. » (Benveniste, PLG1)

Remplacer on par je, donne le sentiment que l'énonciateur parle de sa propre lecture de Freud, or, son intention est de proposer une caractérisation de l'œuvre freudienne qui soit exempte de tout filtrage subjectif, en tout cas présentée comme telle.

(22') " [...], je perçois que le sujet se sert de la parole et du discours pour se "représenter" lui-même tel qu'il veut se voir, tel qu'il appelle l' " autre » à le constater. "

On permet ainsi à l'énonciateur de prétendre que son point de vue est généralisable, ou au contraire, de s'associer à une opinion générale, répandue :

(23) Mais que le mythe lui-même se soit imposé- on sait que les mythes sont utiles, on sait aussi qu'ils conduisent souvent à occulter ou à tronquer la réalité - ne signifie pas pour autant que l'œuvre de Louvet, certaines de ses pièces en tout cas, témoignent comme il l'a été si souvent affirmé des vicissitudes de l'engagement de l'intellectuel ». (Etienne Marest, Lecture de Louvet: suivi d'une bio-bibliographie de Jean Louvet, Lansman, 2002)

Cette analyse ne constitue pas une nouveauté en soi, et elle est parfaitement en accord avec les hypothèses formulées sur le fonctionnement du marqueur par les linguistes énonciativistes, entre autres. Simonin (1984) distingue trois emplois différents de on, qui peut soit être la trace d'un parcours sur la classe des termes sources (valeur générique du pronom), soit être la trace d'une extraction (valeur spécifique mais indéterminée, on peut être remplacé par quelqu'un, par exemple), soit inclure l'énonciateur ou le co-énonciateur. On en contexte, on peut proposer, d'une manière un peu plus synthétique, de voir ce pronom comme la trace de deux opérations de rupture, entre le locuteur et l'énonciateur d'une part, et entre le locuteur et le suet de l'événement, d'autre part, qui aboutit à l'impossibilité de stabiliser la relation entre énonciateur et sujet de l'événement. L'emploi de on offre donc des possibilités référentielles multiples (singulière, collective, incluant ou non l'énonciateur et/ou le co-énonciateur) parmi lesquelles seul le contexte permettra de choisir (et encore). La relation prédicative est alors conçue comme validable par tout terme source, quel qu'il soit en (23) (tout le monde/chacun sait que...) Si l'on ajoute à cela le fait que le locuteur ne se présente pas comme l'énonciateur, c'est-à-dire qu'il prétend ne pas prendre en charge le contenu propositionnel $\left(\mathrm{S}_{1} \omega \mathrm{S}_{0}\right)$ et qu'il n'identifie pas non plus de source énonciative autre, tout se passe alors comme si ce contenu propositionnel ne relevait pas de sa responsabilité, ni de celle de qui que ce soit d'autre, et revêtait une portée factuelle, universelle (ainsi en est-il) qui prévient toute contradiction. On le voit si l'on compare (22) et (22'), puisque contrairement à (22), (22') est contestable dans la mesure où la lecture de Freud est associée d'emblée à une source de subjectivité $\left(\mathrm{S}_{1}=\mathrm{S}_{0}\right)$.

\subsection{On et la deuxième personne}

On met en valeur les propriétés notionnelles du prédicat, et pour cette raison, ne peut être considérer comme l'équivalent strict des pronoms avec lesquels il peut commuter. La substitution de on à un autre pronom entraîne systématiquement des variations 
d'interprétations, même légères. Cela contribue à expliquer le fait que l'emploi de on, dès lors qu'il serait possible d'utiliser un pronom défini ou même un nom propre à la place, ait, comme le relève Muller (1979), nécessairement une portée affective. Mettre en avant la délimitation qualitative de l'occurrence de procès au détriment de sa dimension quantitative lorsque l'événement auquel elle renvoie est effectivement doté d'un ancrage existentiel et le sujet identifié correspond à un choix énonciatif.

C'est le cas par exemple dans l'énoncé suivant, qui évoque la situation d'un joueur de football ayant refusé le traitement de lombalgies par infiltrations :

(24) «Alors comme ça, on a peur des piqûres? 》 (http://www.sofoot.com/alors-comme-caon-a-peur-des-piqures-184999.html)

L'emploi dit hypocoristique de on dans ce type de contexte s'accompagne toujours de la présence d'une adresse, souvent alors, qui invite le co-énonciateur à réagir d'une manière ou d'une autre au propos qui lui est soumis. L'autre caractéristique de ce types d'énoncé est qu'il y est toujours difficile d'identifier la source énonciative. Fløttum, Jonasson et Norén (2007) ont montré comme on pouvait être utilisé dans l'œuvre littéraire (dans Madame Bovary) pour brouiller les frontières entre discours indirect, discours indirect libre et focalisation interne. On permet à l'énonciateur de laisser en suspens la relation de repérage entre $\mathrm{S}_{2}$ par rapport à $\mathrm{S}_{0}$, puisque ces deux termes s'inscrivent en rupture avec $\mathrm{S}_{1}$. Ce faisant, on introduit une forme de brouillage énonciatif. Dans le titre d'article en (24), le ton moqueur est renforcé par le fait que l'on pourrait attribuer au joueur de football la prise en charge d'un énoncé «J'ai peur des piqûres. ", ce qui reviendrait à se moquer de lui. Mais la source énonciative pourrait tout à fait être autre : un individu de l'entourage du joueur, par exemple, un rival, etc. Peu importe. Ce n'est pas la source qui prévaut mais ce qui est dit.

Dans ce type d'énoncés, la formulation du prédicat est souvent connotée affectivement, et les notions de procès font l'objet d'une valuation qui peut être explicite dans la manière même dont le prédicat est formulé :

(25) «Alors comme ça, on veut racailler / on aime la baston / on bouge ses fesses / on aime pas les ornithorynques / on craque pour la petite Belinda, etc. » (www)

Ce type de formulations révèle la mise en valeur des propriétés du procès, l'énoncé véhiculant un commentaire sur le comportement du référent de on (ça va pas la tête, n'importe quoi, ou, au contraire, c'est mignon), ou encore une inadéquation par rapport à un présupposé, comme en (26), qui peut être interprété comme une forme de reproche, dans la mesure où il implique que tout habitant de de la rue Vörösmarty connaît l'Exhortation :

(26) Un jour, vous avez à peine mis le pied dehors que la plaque de votre rue se met à vous dévisager, alors comme ça on habite rue Vörösmarty et on ne connaît pas l'Exhortation? (Nina Yargekov, Double nationalité, POL, 2016)

Dès lors que le référent du sujet est aisément identifiable, le choix d'un pronom qui n'y renvoie pas directement implique nécessairement une visée pragmatique particulière. Il s'agit ici d'attirer l'attention sur les propriétés du prédicat, qui fait systématiquement l'objet d'une valuation.

Avec ces énoncés n'est plus seulement en jeu le repérage entre $S_{2}$ et $S_{1}$ mais aussi celui entre $S_{1}$ et $S_{0}$ puisque l'on n'est pas en mesure d'identifier l'énonciateur de ce qui peut être envisagé comme une forme de discours rapporté, les paroles de l'autre répétées avec plus ou moins de déformation (il paraît que). La rupture entre $\mathrm{S}_{1}$ et $\mathrm{S}_{0}$ intervient aussi dans les emplois génériques de on (cf. supra), en particulier dans des proverbes comme (27) et (28), dans lesquels finalement la relation prédicative est validée quel que soit le terme source, d'une part; et, d'autre part, cette relation prédicative est prise en charge par n'importe quel énonciateur puisqu'il s'agit d'un élément de sagesse populaire, c'est-à-dire par tous et personne à la fois. En tout cas, il n'est pas possible d'y attribuer la prise en charge du contenu propositionnel au seul locuteur :

(27) Ce n'est pas à un vieux singe qu'on apprend à faire des grimaces.

(28) On ne change pas une équipe qui gagne. 
Bien sûr, l'instabilité (qui devient ici universalité) du terme source est ce qui justifie en premier lieu l'emploi de on : dans un proverbe, le prédicat est validé quelle que soit l'identité du référent du sujet. Le contenu propositionnel est aussi le cas quelles que soient les situations d'énonciation et de locution, c'est-à-dire en tout $\mathrm{T}$ et quel que soit $\mathrm{S}$.

Dans cet article, on a proposé un traitement inédit de on puisque nous le considérons comme un pronom de troisième personne. Ce qui fait, à notre sens, sa spécificité par rapport aux autres pronoms personnels, c'est le fait que son emploi instaure d'emblée une rupture entre le locuteur et l'énonciateur, comme si l'on se trouvait dans une situation de discours rapporté dans laquelle l'identité du sujet-point de vue source serait passée sous silence. Une telle analyse permet de rendre compte à la fois de la propension de on à se substituer à n'importe quel pronom du système des personnes du français, mais aussi du fait qu'il ait de nombreux emplois «affectifs » ou «stylistiques », ce qui, à notre avis, est lié à la mise en avant des propriétés du prédicat qu'entraîne l'instabilité référentielle du sujet.

Sur le rapport, avec on, entre locuteur et énonciateur, on aurait sans doute pu développer davantage, en comparant les emplois dans lesquels on est utilisé pour évoquer une rumeur (on dit/raconte/prétend que) avec certaines tournures impersonnelles comme il paraît que, paraît-il et il se dit que. Cela pourra faire l'objet d'une prochaine réflexion.

\section{Références}

Atlani, F. (1984). On l'illusionniste. La langue au ras du texte, 13-29.

Benveniste, E. (1974). Problèmes de linguistique générale, tome 1. Gallimard

Bouscaren, J., \& Chuquet, J. (1987). Grammaire et textes anglais: guide pour l'analyse linguistique. Editions OPHRYS.

Boutet, J. (1986). La référence à la personne en français parlé: le cas de « on ». Langage et société, 38(1), 19-49.

Cabredo Hofherr (2008) «Les pronoms impersonnels humains : syntaxe et interprétation », Modèles linguistiques, 57

Culioli, A. (1990). Pour une linguistique de l'énonciation (PLE), tome 1: Opérations et représentations. Gap, Ophrys.

Culioli, A. (1999). PLE, tome 2: Formalisation et opérations de repérage. Gap, Ophrys.

Culioli, A. (1999). PL3, tome 3 : Domaine notionnel. Gap, Ophrys.

Fløttum, K., Jonasson, K., \& Norén, C. (2007). On: pronom à facettes. De Boeck/Duculot.

François, J. (1984). Analyse énonciative des équivalents allemands du pronom indéfini on.

Kleiber, G.(éd.). Recherches en pragma-sémantique. Paris: Klincksiek, 37-73. 
Grevisse, M. (1986). Le bon usage: grammaire française, avec des remarques sur la langue française d'aujourd'hui. J. Duculot.

Guillaume, G. (1951). La représentation du temps dans la langue française. Langage et science du langage, 193-209.

Guillemin-Flescher, J. (1981). Syntaxe comparée du français et de l'anglais: problèmes de traduction. Editions Ophrys.

Jonasson, K. (2003). Le discours narrativisé dans Une vie de Maupassant et sa traduction dans deux versions suédoises. In La syntaxe raisonnée (pp. 297-308). De Boeck Supérieur.

Landragin, F., \& Tanguy, N. (2014). Référence et coréférence du pronom indéfini on. Langages, (3), 99-115.

Le Goffic, P. (1986). Que l'imparfait n'est pas un temps du passé. Points de vue sur l'imparfait, 55-69.

Maingueneau, D. (2000). Instances frontières et angélisme narratif. Langue française, 74-95.

Muller, C. (1979). Sur les emplois personnels de l'indéfini on. Langue française et linguistique quantitative. Genève, Slatkine, 65-72

Rabatel, A. (2001). La valeur de on pronom indéfini/pronom personnel dans les perceptions représentées. L'information grammaticale, (88), 28-32

Ramat, A. G., \& Sansò, A. (2011). L'emploi indéfini de homo en latin tardif: aux origines d'un «européanisme». La quantification en latin, 93.

Rey-Debove, J. (2001). De on à je vers le nom propre: des pronoms personnels en français.

Bogaards, P., Rooryck, J. et Smith, PJ (éds), Quitte ou double sens. Articles sur l'ambiguïté offerts à Ronald Landheer, Amsterdam: Rodopi, 279-304.

Ruppli, M. (1988). La coordination en français moderne: théorie à l'épreuve d'un corpus. Thèse de Doctorat

Simonin, J. (1984). Les repérages énonciatifs dans les textes de presse. La langue au ras du texte, 133-203.

Skibińska, E. (1984) Traduire en polonais on dans sa spécificité delermienne : une tâche impossible ? Romanica wratislaviensia LI, 83-100.

Tamba-Mecz, I. (1989). La double énigme de on aux concepts de pronom et de personne linguistique en français et en japonais. Sophia linguistica, 27, 5-23.

Tartarin, T. (2013). Le pronom on, marqueur de point de vue ? Etude d'un corpus d'articles de presse français/anglais. In Chuquet, H., Nita, R., \& Valetopoulos, F. Des sentiments au point de vue. 246-268.

Violett, C. (1988). Mais qui est on ? Étude linguistique des valeurs de on dans un corpus oral. LINX, 3, 67-75. 